\title{
Diagnostic reference levels in low- and middle-income countries: Early 'ALARAm' bells?
}

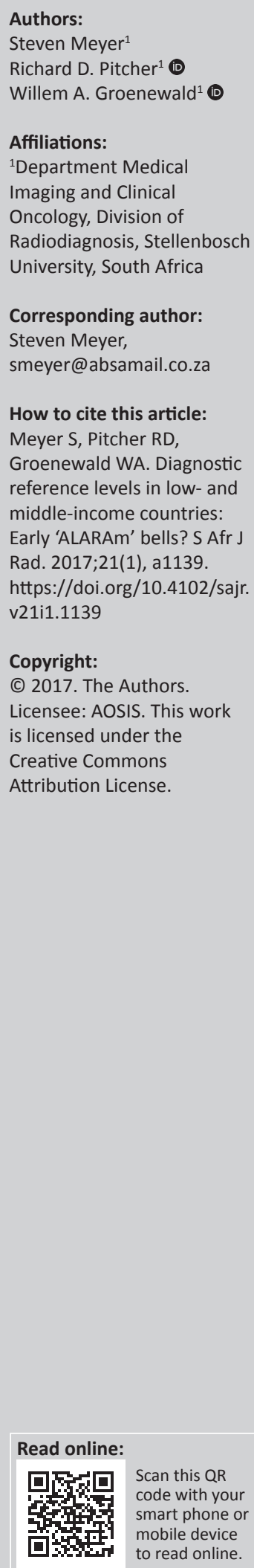

Background: In 1996, the International Commission on Radiological Protection (ICRP) introduced diagnostic reference levels (DRLs) as a quality assurance tool for radiation dose optimisation. Although many countries have published DRLs, available data are largely from high-income countries. There is arguably a greater need for DRLs in low- and middle-income countries (LMICs), where imaging equipment may be older, and trained imaging technicians are scarce. To date, there has been no critical analysis of the published work on DRLs in LMICs. Such work is important to evaluate data deficiencies and stimulate future quality assurance initiatives.

Purpose: To review the published work on DRLs in LMICs and to critically analyse the comprehensiveness of available data.

Material and methods: Medline, Scopus and Web of Science database searches were conducted for English language articles published between 1996 and 2015 documenting DRLs for diagnostic imaging in LMICs. Retrieved articles were analysed and classified by geographical region, country of origin, contributing author, year of publication, imaging modality, body part and patient age.

Results: Fifty-three articles reported DRLs for 28 of 135 LMICs (21\%), reflecting data from 26 of $104(25 \%)$ middle-income countries and 2 of 31 (6\%) low-income countries. General radiography $(n=26,49 \%)$ and computerised tomography $(n=17,32 \%)$ data were most commonly reported. Paediatric DRLs $(n=14,26 \%)$ constituted approximately one-quarter of published work.

Conclusion: Published DRL data are deficient in the majority of LMICs, with the paucity most striking in low-income countries. DRL initiatives are required in LMICs to enhance dose optimisation.

Note: A selection of conference abstracts: RSSA/SASPI Paediatric Imaging Congress, 03-06 November 2016, Spier Estate, Stellenbosch, Note: A selection of conference abstracts: RSSA/SASPI Paediatric Imaging Congress, 03-06 November 2016, Spier Estate, Stellenbosch, South Africa. Faculty collaborators: Professor Kassa Darge (Body Imaging, University of Pennsylvania, Philadelphia, USA), Professor Edward Lee (Thoracic Imaging, Harvard University, USA), Professor Beverley Newman (Cardiac Imaging, Stanford University, California, USA), Professor Kimberly Applegate (Image Gently and Body Imaging, Emory University, Atlanta, USA) and Professor Savvas Andronikou (Thoracic Imaging, University of Bristol, UK) supported by South African Paediatric Radiologists, co-ordinated by Dr Jaishree Naidoo, President of the African Society of Paediatric Imaging and Head of Division of Paediatric Radiology, Charlotte Maxeke Johannesburg Academic Hospital. 